\title{
EVALUATION OF DRIVER'S ECO-DRIVING SKILLS BASED ON FUZZY LOGIC MODEL - A REALISTIC EXAMPLE OF VEHICLE OPERATION IN REAL-WORLD CONDITIONS
}

\author{
Marko Stokic*, Vladimir Momcilovic, Davor Vujanovic \\ University of Belgrade - Faculty of Transport and Traffic Engineering,Serbia
}

It is well known that fuzzy logic is a processing tool in circumstances lacking of clear linguistic information, as well as making conclusions based on imprecise assertions and rough data. Eco-driving rules that the drivers should comply with are not always made of concrete values (exact acceleration / deceleration rates, torque or headway / distance kept from the vehicle ahead, etc.), but often linguistically expressed and subjective (e.g. soft acceleration, mid-range engine speed, soft deceleration, sufficient distance, etc.). Therefore, the authors recognized fuzzy logic potentials as an efficient tool to overcome all mentioned barriers and thus to increase vehicle energy eficiency and reduce emissions of harmful gases which are main goals of eco-driving. The primary objective of this paper is to raise the awareness on the potentials and efficiency of fuzzy logic systems' use in eco-driving as a tool for achieving more ecologically \& economically sustainable road transport. The rules that drivers should follow in order to achieve and maintain eco-driving goals, as well as the parameters to be monitored to evaluatedriver's behaviour i.e. the compliance with eco-driving rules are presented in the paper. The authors propose a driver rating system based on the fuzzy logic model constructed within MatLab. Within the proposed model the input parameters are actual acceleration/deceleration rates, engine speed and accelerator pedal pressure(APP) and while model output are driver ratings (scores ranging from 0 to 10 points) after completed a driving cycle. A real-world example based on data collected via vehicle OBDII connector by a TEXA logging device in realistic vehicle operation conditions. The consequent actual results of drivers' behaviour rating tool based on the proposed model are presented in the paper.

Key words: Eco-driving, Support, Fuzzy logic, Driver rating system, Vehicle energy efficiency, Tools

\section{INTRODUCTION}

Transport related environmental pollution is a significant problem and subject of various studies and research in the past three decades. The aim is to reduce harmful exhaust gas emissions from vehicles, providing that it doesn't affect the realization of the transport process. One of the solutions is environmentally friendly driving, more popularly called eco-driving. Eco-driving is the way in which drivers by applying certain strategies and behaviour rules can reduce environmental pollution by greenhouse gas emissions while driving [1].

Beloufaet al. [2] define eco-driving as a concept used to achieve vehicle energy efficiency, or as a driving strategy that aims to reduce fuel consumption on the same trip distances. Momčilović et al. [3] pointed out that eco-driving is an environmentally responsible and cost-effective driving method, which implies a safe and responsible driving technique with minimal fuel consumption, as well as a driving styleincluding active use of modern technological solutions adapted to modern vehicles. As the fuel cost in road transport makesbetween $30 \%$ and $50 \%$ of total costs [4], eco-driving can have a significant impact on the profit of the observed companies through fuel consumption reduction, and therefore has an impact on pollution reduction. In this sense, eco-driving is a kind of "win-win" situation for solving the mentioned problem. According to Forset al.[1], eco-driving can result in fuel savings up to $27 \%$ if applied on trucks, while according to Young et al. [5]it can save between 5 and 15\%. Barkenbus[6] states that possible savings in case of individual passenger cars are between 5 and $10 \%$. Based on aliterature review it can be concluded that estimated fuel consumption savings are at similar levels when eco-driving is applied.

Since the nature of principles and techniques applied while driving in order to reduce fuel consumption is linguistic and vaguely defined, fuzzy logic has found its application in eco-driving rules. Fuzzy logic is a type of logic that includes more than just true or false values, it is the logic that deals with situations where one cannot give a clear yes/no (true/false)answer [7]. Fuzzy logic is a system of reasoning and computation in which the objects of reasoning and computation are classes with unsharp (fuzzy) boundaries [8].The fuzzy logic allows the processing of fuzzy data, as well as the adoption of certain conclusions based on imprecise assertions and inaccurate data [9]. Fuzzy logic has been successfully used for various problems such as evaluation of services in a manufacturing company [10], as support in industrial engineering management [11].Also,there are several papers in the literature where authors applied fuzzy logic on eco-driving in order to achieve a more economical and environmentally friendly driving [12],[13],[14],[15].

In order to have active driver support system in view of eco-driving, it is necessary to clearly define parameters 
affecting the fuel consumption and timely correct them either by advices of eco-driving instructor or by given signals fromon-board eco-driving support systems and devices.

The paper is structured as follows. Chapter 2 comprises a literature review regarding eco-driving principles and techniques to be applied while driving and parameters to be monitored in order to evaluate driver's behaviour. In Chapter 3 the fuzzy logic model developed by the authors is presented and used to evaluate aforementioned driver's behaviour regarding eco-driving principles and techniques. The fuzzy logic model testing is conducted and the results are presented in Chapter 4, while some conclusions and future research are given in chapter 5 .

\section{PRINCIPLES AND TECHNIQUES OF ECO-DRIVING}

Based on the literature review, in this chapter will be presented the principles and techniques of eco-driving as well as relevant parameters which should be monitored.

In that sense, Fors et al. [1] observed velocity, acceleration, deceleration, inertia movement on the longitudinal slope (downhill), overspeeding, recommendations on speed adjustment to new speed limits traffic lights as parameters in their research related to heavy goods vehicles.

On the other hand, Beloufa et al. [2], during the research on the simulator, took into consideration 6 eco-driving rules, including: changing gear before 2500 RPM, driving in the highest possible gear, maintaining a constant speed, traffic anticipation, established deceleration principle,and switching off the engine in situations where it is expected for vehicle to idle more than 30 seconds. Sanguinetti et al. [16] observe six classes of eco-driving rules: vehicle handling, cabin comfort, travel planning, cargo handling, fuel supplying, and vehicle maintenance. For the purposes of this paper, only the first class, i.e. the rules of eco-driving to be applied while driving are given. In that sense, in this class, Sanguinetti et al. [16] classify acceleration, the use of cruise control, deceleration, idling and the selection of the appropriate parking place - activities which should conduct before switching off the engine. Also Sanguinetti et al. [16] emphasized that accelerator pedal should never be pressed more than half in order to avoid aggressive acceleration (acceleration which requires high and medium power) and that the standard deviation of accelerator pedal pressure should be measured (the smaller the standard deviation, the more energy efficient the driving). In addition, it must be ensured that the engine speed never exceeds 3500 RPM, as well as that it is necessary to switch to a higher gear when the engine speed is between 2000 and 2500 RPM. Regarding the engine speed Araujo et al. [12] state that the driver should switch to a higher gear before the engine speed reaches 2500 RPM in the case of petrol vehicles, and before 2000 RPM in case of diesel powered vehicles.Also, it is necessary to maintain a constant speed at the highest possible gear because that is the most energy efficient [16]. Like Beloufa et al. [2] and Sanguinetti et al. [16] emphasize it is necessary to maintain the distance to the vehicle ahead to use inertia and avoid unnecessary use of brakes. In this sense, the driver should apply engine braking, also advised while decelerating in addition to classical braking.

Beusen et al. [17] carried out a 10-month study in real-world conditions involving 10 drivers, taking into consideration the distance traveled, engine speed, accelerator pedal pressure, current and average fuel consumption, average speed, average engine speed when the driver switches to a higher gear (taken value is between 2000 and 2500 RPM), percentage of time vehicle moves in certain gearunder inertia, its fuel consumption being equal to 0 liters per $100 \mathrm{~km}$, percentage of time vehicle accelerates or decelerates rapidly, percentage of idling time, percentage of distance traveled at optimal engine speed (between 1100 and 1700 RPM), as well as percentage of distance traveledat speeds greater than $120 \mathrm{~km} / \mathrm{h}$.

Gilman et al. [18] observed the following parameters during driver's behaviour assessment: percentage of time at certain speed intervals, percentage of time at certain engine speed intervals, speed oscillations (local maximum and minimum), percentage of time when speed and acceleration product is between $3-10 \mathrm{~m}^{2} / \mathrm{s}^{3}$; percentage of time when acceleration is greater than 2.5 $\mathrm{m} / \mathrm{s}^{2}$, relatively positive acceleration and average deceleration.

Several authors [16],[2],[19] state it is necessary to turn off the engine if longer idling is expected. In that sense, Beloufaet al. [2] define the requirement for turning off the engine for idling longer than 30 seconds, Sanguinetti et al. [16] define the same requirement for idling longer than one minute, while Diaz-Ramirez et al.[19]state that the engine should be turned off for more than 5 minutes of idling. Diaz-Ramirez et al. [19] observed also the following parameters: driving errors(e.g. excessive speed, acceleration, and braking), trip conditions (e.g. trip length and road topography), driver behaviour (e.g. enginespeed in revolutions per minute [RPM] and vehicle speed $[\mathrm{km} / \mathrm{h}]$ ), driver profile (e.g. age, experience, and level of education) and operational needs (e.g. load and vehicle attributes).

Pozueco et al. [13] observed parameters such as vehicle speed, engine speed, location (GPS), current fuel consumption, acceleration and other parameters that can be read from the Engine Control Unit. Araujo et al. [12] considered parameters such as speed, acceleration, altitude, signal from accelerator pedal, fuel consumption, engine speed, and idling. Cvetkovic et al. [20] examined the engine speed, vehicle speed, acceleration and deceleration. In addition, terrain configuration has considerable impact on fuel consumption, specifically uphill climbing and downhill efficiency. Sanguinetti et al. [16] consider necessary to accelerate the vehicle additionally before the uphill and releasing the accelerator pedal 
while riding downhill.

Based on literature review, it is concluded that there are many principles and techniques that should be respected and parameters to be considered in order to achieve eco-efficient driving. Although parameter values are deterministic and relatively easy to establish with the assistance of adequate devices, eco-driving principles and techniques based on these parameters are not clearly defined, and the exact limits of parameters are not strictly distinguishedand fuzzy. A specific problem arises when defining rules taking into account two or more mutually dependent parameters, aside fromthe linguistic nature of certain rules (accelerate mildly, gently, with medium acceleration, as fast as possible to the desired speed, etc.). Consequently, fuzzy logic becomesmore and more important in the designing of fleet management support systems and on-board eco-driving support devices. The fuzzy model for evaluating driver'sbehaviour is described in detail in the following chapter.

\section{PROPOSED FUZZY MODEL}

A model for evaluating driver's behaviour during driving is presented hereafter. The authors develop a Multiple Inputs Single Output (MISO) fuzzy logicmodel.MatLab 2017a was used to create the fuzzy logic model. Based on the literature review presented in Chapter 2 and- long-term expertise, the authors have adopted the following input parameters in the developed model: engine speed in revolutions per minute (RPM), acceleration/ deceleration rate and accelerator pedal pressure (APP). The model is developed in a general form and allows to be used both on diesel and petrol powered vehicles. On the basis of values of the above mentioned three input parameters, the model produces driver's average rating as output (in interval from 0 to 10 points). Membership functions (MF)are presented in the following figures.

As shown in Figure 1, the input parameter „Engine revolutions per minute" is defined with three membership functions: small, medium and big engine speed in revolutions per minute. Acceleration/deceleraton is considered through 5 MF's, where 2 MF's define big and medium deceleration (negative acceleration), 2 MF's defines big and medium acceleration and $1 \mathrm{MF}$ involves both small accelerations and decelerations (Figure 2).

Accelerator pedal pressure is defined with three MF's small, medium and high.

Regarding output values, $5 \mathrm{MF}$ 'sare defined on the interval from 0 to 10 points representing very bad, bad, average, good and very good driver ratings when analyzing driving performance based on previous parameters. Based on the observed input parameters and the corresponding MF's, 45 rules are defined where several

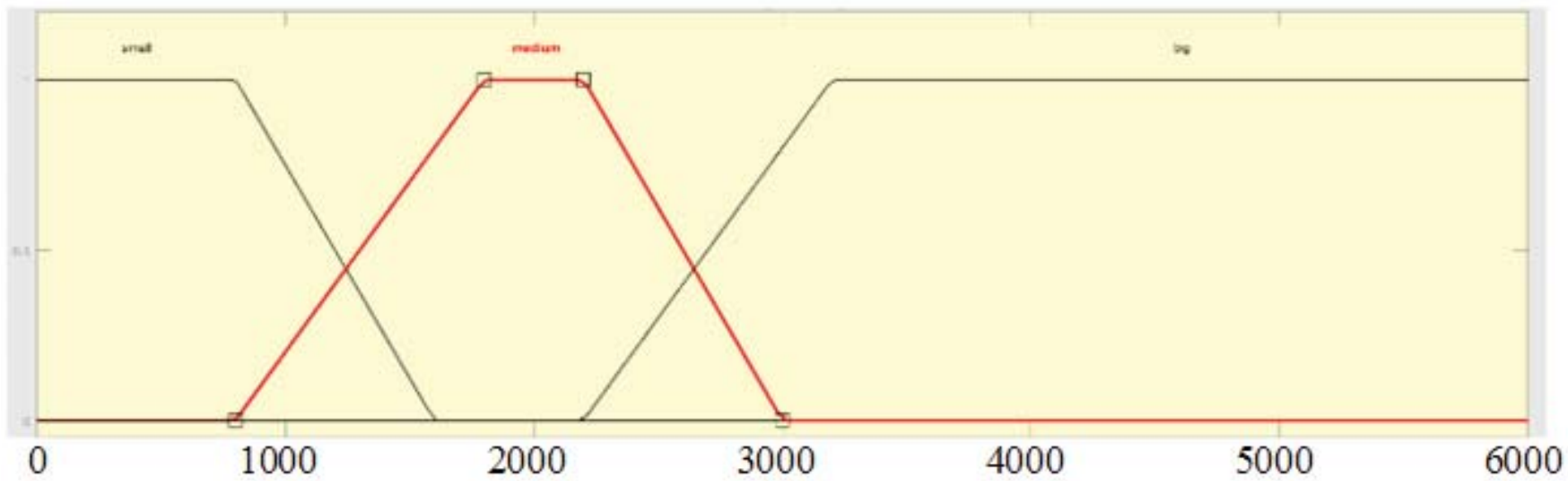

Figure 1: Membership functions for input 1 - Engine revolutions per minute

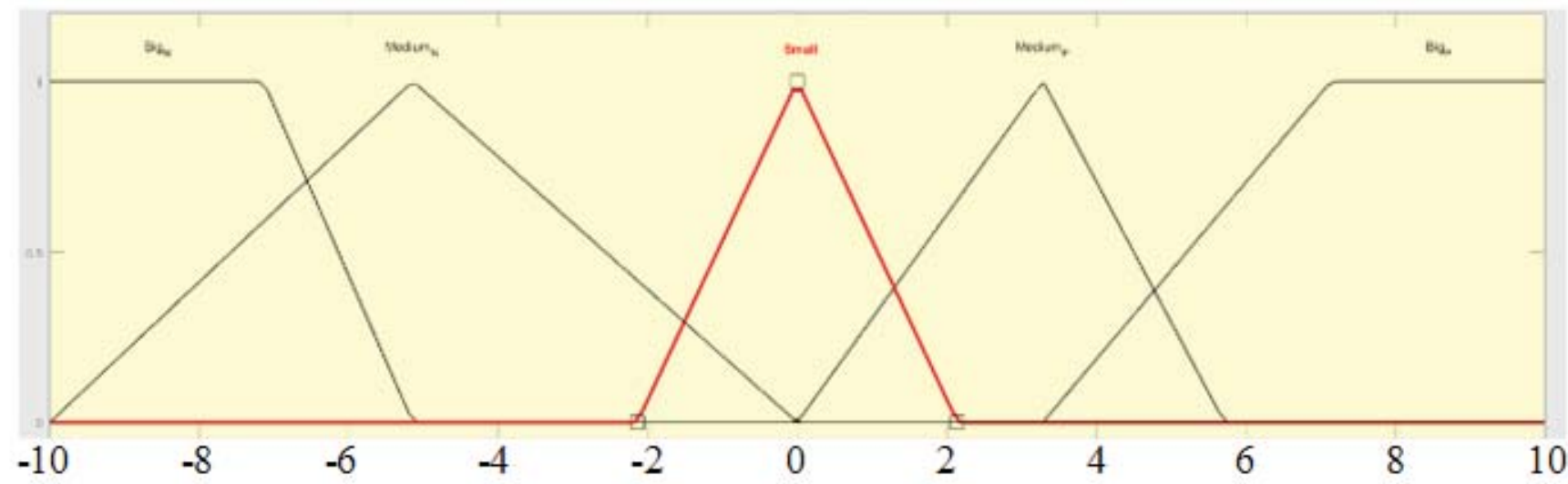

Figure 2: Membership functions for input 2 - Acceleration/deceleration 


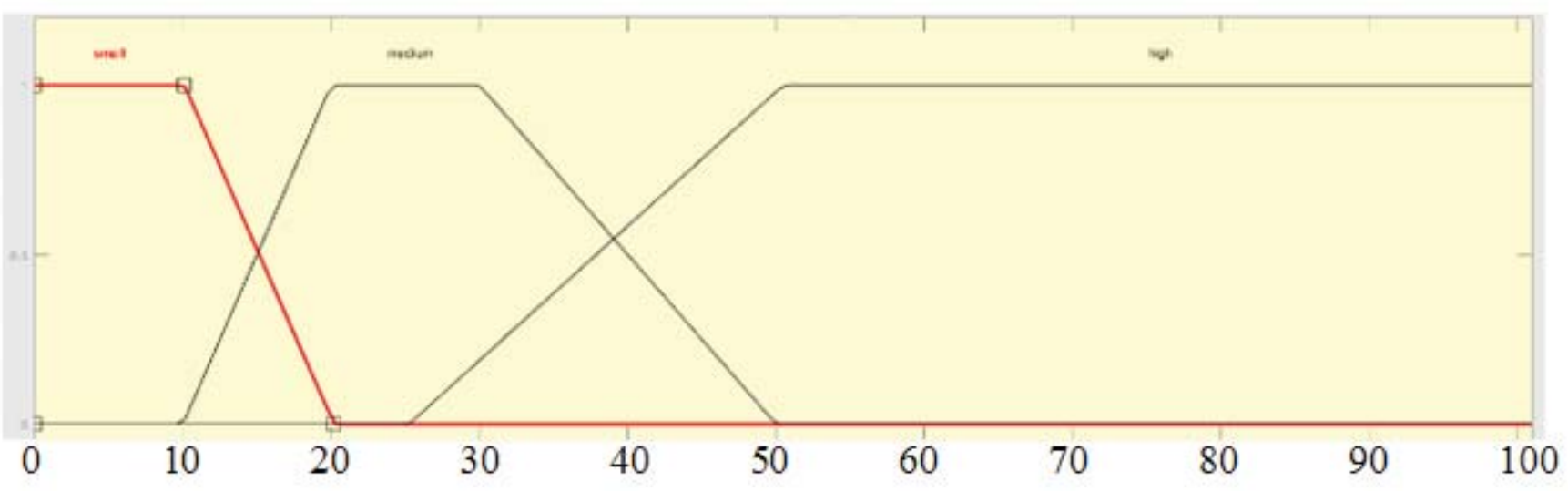

Figure 3: Membership functions for input 3 -Accelerator pedal pressure

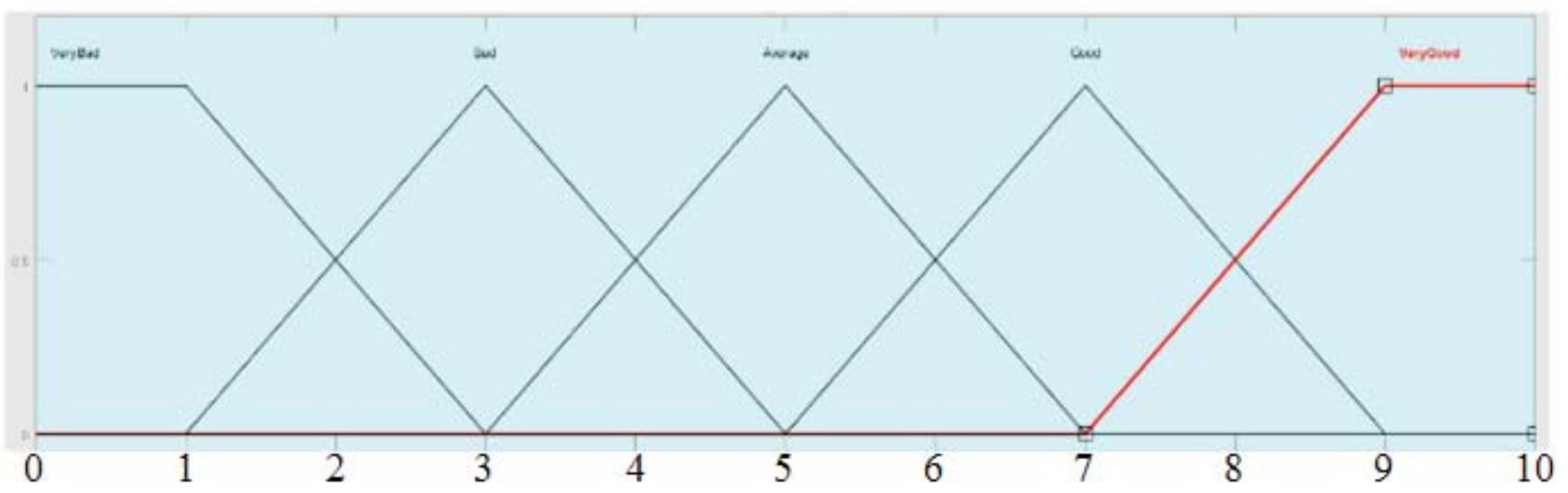

Figure 4: Membership functions for output - Driver ratings

Table 1: Fuzzy rules

1. $(\mathrm{RPM}==$ small $) \&($ Acceleration==Small $) \&(\mathrm{APP}==\mathrm{Small})=>$ (output $1=$ VeryGood $)(1)$

2. $(\mathrm{RPM}==$ small $) \&($ Acceleration $==$ Small $) \&(\mathrm{APP}==$ Medium $)=>$ (output $1=\mathrm{Good})(1)$

3. $(\mathrm{RPM}==$ small $) \&($ Acceleration $==$ Small $) \&(\mathrm{APP}==\mathrm{Big})=>$ (output1=Average $)(1)$

4. $(\mathrm{RPM}==$ high $)$ \& (Acceleration==MediumPositive $) \&(\mathrm{APP}==$ Medium $)=>($ output1=Bad $)(1)$

5. $(\mathrm{RPM}==$ high $) \&($ Acceleration==MediumNegative $) \&(\mathrm{APP}==\mathrm{Big})=>($ output1=VeryBad $)(1)$

are shown as example in Table 1. For the formulation of rules the "and" connection was used as follows: If Input 1 is linguistic variable and Input 2 is linguistic variable and Input 3 is linguistic variable then Output is linguistic variable. For the defuzzification process, centroid method was used.

\section{TESTING OF DEVELOPED FUZZY MODEL}

The testing of developed fuzzy model was carried out with passenger cars Renault Laguna powered with a 1.9 diesel engine (production year 2006), and Suzuki Vitara powered with a 1.6 petrol engine (production year
2017). The TEXA MATRIX OBDII connector was used to monitor the parameters which are used as inputs in the fuzzy logic model (Figure 5). Also, the device records observed parameters in specified time interval $(0.25,0.5$ or 1 second). During device configuration on TEXA software, recording of mentioned parameters was selected, and it was chosen to collect data at the frequency of $1 \mathrm{~Hz}$ (one value per second). After each run, the data was transferred from the device (1) to TEXA software installed on the computer (2) using the base station (3), as shown in Figure 5. For the needs of testing the developed fuzzy logic model, 10 test runs were carried out with mentioned vehicles under different operating con 


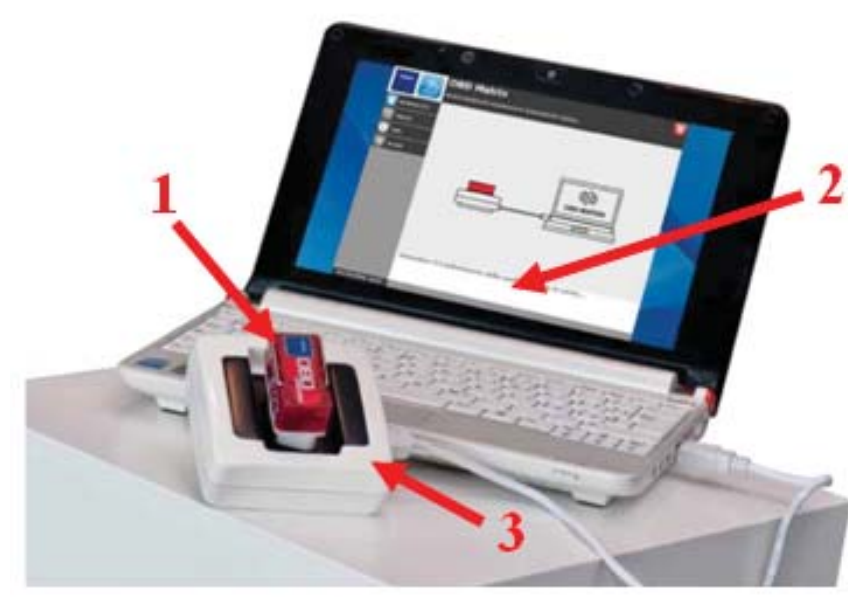

Figure 5: TEXA MATRIX device

ditions (urban, suburban and extraurban) and different driving styles (passive, aggressive and combined). The test results of the developed fuzzy logic modelare shown in Table 2. During each run, the drivers operated the vehicles with different driving styles (passive, combined, aggressive) under different operating conditions (urban, suburban, and extraurban) without additional load (no passengers or cargo). Different driving styles (passive, combined and aggressive) are simulated to determine the sensitivity of the developed model. In cases of more aggressive driving style, the developed model should, as an output, give a lower driver rating than in case of passive driving style for the same operating conditions. As shown from the results presented in Table 2, the best rating with the Suzuki Vitara was achievedwith a passive driving style amounting 8.19 , while the driver rating for aggressive driving was 7.51 for the same car. The difference in driver's ratings while driving follows the difference in fuel consumption. In this sense, the higher the driver's rating the lower the fuel consumption, and opposite, the lower the driver's rating the higher the fuel consumption.

When analyzing the Renault Laguna passenger car data, it can be concluded that there are differences in the obtained ratings although driving distances are short. Even with this vehicle and in suburban operation conditions, the fuzzy logic model shown its sensitivity. The aggressive driving style has lower ratings compared to the combined driving style, especially when the passive driving style is observed in the same operating conditions. The above mentioned difference in the driver's ratings implies a difference in the fuel consumption. Thus, for passive driving style, the driver's rating is 8.66 , and the average fuel consumption is 5.4 litersper $100 \mathrm{~km}$, while for aggressive driving behaviour, the driver's rating is 8.17 , corresponding to an average fuel consumption of $9.3 \mathrm{li}$ ters per $100 \mathrm{~km}$. In addition, it should be emphasized that the presented fuzzy logic model is general model developed for both diesel and petrol powered vehicles. Since diesel powered vehicles operate at lower engine speeds, the more aggressive driving style of diesel cars consists in engine speeds more suitable for operation of petrol powered vehicles. Consequently, driver's ratings with Renault Laguna are slightly better than the second one with a Suzuki Vitara. Besides that, Renault Laguna was driven in more favourable operating conditions (suburban and extraurban) than Suzuki Vitara (only urban). Other factors that can influence the ratings are the driving distances. With higher mileage, the data should be more precise, which is the planned task for future research. However, on the basis of tests, it can be concluded that the developed fuzzy logic model is sensitive to different driving styles and it can serve as decision support system for fleet managers in the assessment of the driver's performance. According to tests it can also be concluded that the developed fuzzy logic model could

Table 2: Results of driver rating fuzzy model testing

\begin{tabular}{|c|c|c|c|c|c|c|}
\hline Run No. & Vehicle & $\begin{array}{c}\text { Route dist. } \\
{[\mathrm{km}]}\end{array}$ & $\begin{array}{c}\text { Operation } \\
\text { conditions }\end{array}$ & Driving style & $\begin{array}{c}\text { Fuel con- } \\
\text { sumption } \\
/ / 100 \mathrm{~km}\end{array}$ & $\begin{array}{c}\text { Average } \\
\text { rating }\end{array}$ \\
\hline 1 & Suzuki Vitara & 15 & Urban & Aggressive & 7.5 & 7.51 \\
\hline 2 & Suzuki Vitara & 15 & Urban & Combined & 7.3 & 7.58 \\
\hline 3 & Suzuki Vitara & 15 & Urban & Combined & 7.0 & 7.70 \\
\hline 4 & Suzuki Vitara & 15 & Urban & Passive & 6.0 & 8.19 \\
\hline \multicolumn{7}{|l|}{} \\
\hline 1 & Renault Laguna & 3 & Suburban & Passive & 5.4 & 8.66 \\
\hline 2 & Renault Laguna & 4.3 & Suburban & Combined & 7.2 & 8.31 \\
\hline 3 & Renault Laguna & 4.6 & Suburban & Combined & 7.5 & 8.59 \\
\hline 4 & Renault Laguna & 5.1 & Suburban & Passive & 6.8 & 8.55 \\
\hline 5 & Renault Laguna & 3.7 & Suburban & Aggressive & 9.3 & 8.17 \\
\hline 6 & Renault Laguna & 56 & Extraurban & Aggressive & 6.0 & 8.35 \\
\hline
\end{tabular}


have impact on reducing fuel consumption in transport and logistics companies. For further applicability testing the developed fuzzy logic model will be implemented in at least one transport company with a vehicle fleet to demonstrate potential fuel consumption savings.

In that sense, for the purposes of calculating the driver's average ratings, basic version of the code in MatLab 2017awas made which can be used as a decision support system to fleet managers or people who want to evaluate their own driving style. The code consists in observed inputs, their membership functions, fuzzy logical rules giving as output average driver'srating related to his/her behaviour and performance for each run driving style in every vehicle operating condition and for every vehicle load capacity utilization.

\section{CONCLUSIONS AND RECOMMENDATIONS}

The aim of this paper is to show the importance of using the fuzzy logic in eco-driving practice, and therefore in increasing the vehicle energy efficiency. The paper presents eco-driving principles and techniques that drivers should follow in order to achieve and maintain eco-driving goals, as well as the parameters to be monitored to assess drivers. The authors developed a driver rating system based onfuzzy logic model in order to evaluate driver's behaviour. The fuzzy logic model (with some modifications) can be used as a general evaluation system of drivers in transport and logistics companies. The developed model was tested and it's sensitivity for different driving styles (passive, combined, aggressive) but same vehicle, driver, operating conditions (urban, suburban, extraurban) and vehicle load was determined. Based on the testing, the aggressive driving style has lower ratings compared to the combined driving style, especially when the passive driving style is observed in the same operating conditions. It was found that the higher the driver's rating the lower the fuel consumption, and opposite, the lower the driver's rating the higher the fuel consumption (in liters per $100 \mathrm{~km}$ ). In this sense, the developed model serves to fleet managers in transport and logistics companies as a driver evaluation support system and as a measure for better implementation of eco-driving, i.e. to increase the vehicle fleet energy efficiency. Developed model can also serve to individual passenger car users as a support to increase the energy efficiency of their vehicles.

Within the directions of the future research, it is necessary to adjust developed fuzzy logic model to different vehicle categories, fuels/propulsions, operating conditions, and vehicle loads. Also, directions of future research involve its implementation in one or more transport and logistics companies in the Republic of Serbia and evaluation of its application through total fuel consumption savings.

\section{ACKNOWLEDGMENT}

The paper is partially supported by the Ministry of Education, Science and Technological Development of the Republic of Serbia through project TR36010. Also the authors wish to express their gratitude to the company "Marinković HOFMANN" forthe support in the research and for providing the TEXA MATRIX device for real-time data collection in real-world conditions.

\section{REFERENCES}

1. Fors, C., Kircher, K., Ahlstrom, C.(2015).Interface design of eco-driving support systems - Truck drivers' preferences and behavioural compliance. Transportation Research Part C, vol. 58, 706-720, DOI: 10.1016/j.trc.2015.03.035

2. Beloufa, S., Cauchard, F., Vedrenne, J., Vailleau, B., Kemeny, A., Merienne, F., Boucheix, J. M.(2017). Learning eco-driving behaviour in a driving simulator: Contribution of instructional videos and interactive guidance system. Transportation Research Part F, vol. 61, 201-216,DOI: 10.1016/j.trf.2017.11.010

3. Momčilović, V., Dimitrijević, B., Stokić, M.(2017). Eco-driving - potentials and opportunities within green logistics. Proceedings of the 3rd Logistics International Conference LOGIC 2017, Belgrade, Serbia, 222-227

4. Momčilović, V., Cvetković, M.(2011).Koncept obuke vozača za ekološku vožnju.Proceedings of conference"Ka održivom transportu 2011", 79-90

5. Young, M.S., Birrell, S.A., Stanton, N.A.(2011). Safe Driving in green world: A review of driver performance benchmarks and technologies to support "smart" driving. Applied Ergonomics, vol. 42, no. 4, 533-539, DOI: 10.1016/j.apergo.2010.08.012

6. Barkenbus, J.N.(2010). Eco-driving: An overlooked climate change initiative. Energy Policy, vol. 38, no. 2, 762-769, DOI: 10.1016/j.enpol.2009.10.021

7. Mendel, J.M.(2017). Uncertain Rule-Based Fuzzy Systems: Introduction and New Directions 2nd Edition.Springer International Publishing, DOI: 10.1007/978-3-319-51370-6

8. Zadeh, L. (2015). Fuzzy logic - a personal perspective. Fuzzy Sets and Systems, vol. 281, 4-20, DOI: 10.1016/j.fss.2015.05.009

9. Teodorović, D., Šelmić, M. (2012).Računarska inteligencija u saobraćaju.University of Belgrade, Faculty of Transport and Traffic Engineering

10. Oblak, L., Kuzman, M.K., Grošelj, P.(2017). A fuzzy logic-based model for analysis and evaluation of services in a manufacturing company. Journal of Applied Engineering Science, vol. 15, no. 3, 258-271, DOI: 10.5937/jaes15-13399 
11. Nunes, I.L.(2012). Fuzzy systems to support industrial engineering management. Journal of Applied Engineering Science, vol. 10, no. 3, 143-146, DOI: 10.5937/jaes10-2510

12. Araujo, R., Igreja, A., de Castro, R., Araujo, R. E. (2012). Driving Coach: a Smartphone Application to Evaluate Driving Efficient Patterns.Proceedings of2012 Intelligent Vehicle Symposium, Spain1005-1010

13. Pozueco, L., Pañeda, X. G., Tuero, A. G., Díaz, G., García, R., Melendi, D., Pañeda, A. G., Sánchez, J. A.(2017). A methodology to evaluate driving efficiency for professional drivers based on a maturity model. Transportation Research Part C, vol. 85, 148-167, DOI: 10.1016/j.trc.2017.09.017

14. Chou, W-Y., Lin, Y-C., Lin, Y-H., Chen, S-Y. (2012). Intelligent eco-driving suggestion system based on vehicle loading model. Proceedings of 12th International Conference on ITS Telecommunications, 558562, DOI: 10.1109/ITST.2012.6425241

15. Massoud, R., Poslad, S., Bellotti, F., Berta, R., Mehran, K., De Gloria, A. (2018). A Fuzzy Logic Module to Estimate a Driver's Fuel Consumption for Reality-Enhanced Serious Games. International Journal of Serious Games, vol. 5, no. 4, 45-62, DOI: 10.17083/ijsg.v5i4.266

16. Sanguinetti, A., Kurani, K., Davies, D.(2017). The many reasons your mileage may vary: Toward a unifying typology of eco-driving behaviors. Transportation Research Part D, vol. 52, 73-84, DOI: 10.1016/j. trd.2017.02.005
17. Beusen, B., Broekx, S., Denys, T., Beckx, C., Degraeuwe, B., Gijsbers, M., Scheepers, K., Govaerts, L., Torfs, R., Panis, L. I.(2009). Using on-board logging devices to study the longer-term impact of an eco driving course. Transportation Research Part D, vol. 14, no. 7, 514-520, DOI: 10.1016/j.trd.2009.05.009

18. Gilman, E., Keskinarkaus, A., Tamminen, S., Pirttikangas, S., Roning, J., Riekki, J.(2015).Personalised assistance for fuel-efficient driving. Transportation Research Part C, vol. 58, 681-705, DOI: 10.1016/j. trc.2015.02.007

19. Díaz-Ramirez, J., Giraldo-Peralta, N., Flórez-Ceron, D., Rangel, V., Mejía-Argueta, C., Huertas, J. I., Bernal, M.(2017).Eco-driving key factors that influence fuel consumption in heavy-truck fleets: A Colombian case. Transportation Research Part D, vol. 56, 258270, DOI: 10.1016/j.trd.2017.08.012

20. Cvetković, M., Momčilović V., Dimitrijević, B.(2015). Performance Indicators For Professional Drivers' Evaluation In Supply Chain.Proceedings of 2nd Logistics International Conference LOGIC 2015, Belgrade, Serbia, 253-258 